\title{
Analysis of prognostic genes in the tumor microenvironment of lung adenocarcinoma
}

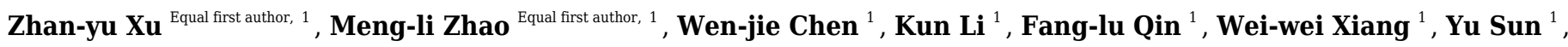 \\ Jiang-bo Wei ${ }^{1}$, Li-qiang Yuan ${ }^{1}$, Shi-kang Li ${ }^{\text {Corresp., }}{ }^{1}$, Sheng-hua Lin ${ }^{\text {Corresp. } 1}$ \\ ${ }^{1}$ Department of Thoracic and Cardiovascular Surgery, The First Affiliated Hospital of Guangxi Medical University, Nanning, China \\ Corresponding Authors: Shi-kang Li, Sheng-hua Lin \\ Email address: 201013064@qq.com, weipeng@stu.gxmu.edu.cn
}

Background: Prognostic genes in the tumor microenvironment play an important role in immune biological processes and the response of cancer to immunotherapy. Thus, we aimed to assess new biomarkers that are associated with immune/stromal cells in lung adenocarcinomas (LUAD) using the ESTIMATE algorithm, which also significantly affects the prognosis of cancer. Methods: The RNA sequencing (RNA-Seq) and clinical data of LUAD were downloaded from the the Cancer Genome Atlas (TCGA). The immune and stromal scores were calculated for each sample using the ESTIMATE algorithm. The LUAD gene chip expression profile data and the clinical data (GSE37745, GSE11969, and GSE50081) were downloaded from Gene Expression Omnibus (GEO) for subsequent validation analysis. Differentially expressed genes were calculated between high and low score groups. Univariate Cox regression analysis was performed on differentially expressed genes (DEGs) between the two groups to obtain initial prognosis genes. These were verified by three independent LUAD cohorts from the GEO database. Multivariate Cox regression was used to identify overall survival-related DEGs. UALCAN and the Human Protein Atlas were used to analyze the mRNA /protein expression levels of the target genes. Immune cell infiltration was evaluated using the Tumor Immune Estimation Resource (TIMER) and CIBERSORT methods, and stromal cell infiltration was assessed using xCell. Results: In this study, immune scores and stromal scores are significantly associated with the clinical characteristics of LUAD, including T stage, M stage, pathological stage, and overall survival time. 530 DEGs (18 upregulated and 512 downregulated) were found to coexist in the difference analysis with the immune scores and stromal scores subgroup. Univariate Cox regression analysis showed that 286 of the 530 DEGs were survival-related genes $(p<0.05)$. Of the 286 genes initially identified, nine prognosis-related genes (CSF2RB, ITK, FLT3, CD79A, CCR4, CCR6, DOK2, AMPD1, and IGJ) were validated from three separate LUAD cohorts. In addition, functional analysis of DEGs also showed that various immunoregulatory molecular pathways, including regulation of 
immune response and the chemokine signaling pathways, were involved. Five genes (CCR6, ITK, CCR4, DOK2, and AMPD1) were identified as independent prognostic indicators of LUAD in specific data sets. The relationship between the expression levels of these genes and immune genes was assessed. We found that CCR6 mRNA and protein expression levels of LUAD were greater than in normal tissues. We evaluated the infiltration of immune cells and stromal cells in groups with high and low levels of expression of CCR6 in the TCGA LUAD cohort. In summary, we found a series of prognosisrelated genes that were associated with the LUAD tumor microenvironment. 
3 Zhan-yu Xu ${ }^{1 \#}$, Meng-li Zhao ${ }^{1 \#}$, Wen-jie Chen ${ }^{1}$, Kun Li ${ }^{1}$, Fang-lu Qin ${ }^{1}$, Wei-wei Xiang ${ }^{1}, Y u$

4 Sun ${ }^{1}$, Jiang-bo Wei ${ }^{1}$, Li-qiang Yuan ${ }^{1}$, Shi-kang Li ${ }^{1 *}$, Sheng-hua Lin ${ }^{1 *}$

5 1. Department of Thoracic and Cardiovascular Surgery, The First Affiliated Hospital of Guangxi

6 Medical University, Nanning, Guangxi Zhuang Autonomous Region 530021, P. R. China.

7 *Corresponding author:

8 Shi-kang Li,

9 Department of Thoracic and Cardiovascular Surgery, The First Affiliated Hospital of Guangxi

\section{Analysis of prognostic genes in the}

\section{tumor microenvironment of lung adenocarcinoma}

Medical University, Nanning, Guangxi Zhuang Autonomous Region 530021, P. R. China.

E-mail address: 1sksci10@163.com

Sheng-hua Lin,

Department of Thoracic and Cardiovascular Surgery, The First Affiliated Hospital of Guangxi

Medical University, Nanning, Guangxi Zhuang Autonomous Region 530021, P. R. China.

E-mail address: weipeng@stu.gxmu.edu.cn

\section{ABSTRACT}

Background: Prognostic genes in the tumor microenvironment play an important role in immune biological processes and the response of cancer to immunotherapy. Thus, we aimed to assess new biomarkers that are associated with immune/stromal cells in lung adenocarcinomas (LUAD) using the ESTIMATE algorithm, which also significantly affects the prognosis of cancer.

Methods: The RNA sequencing (RNA-Seq) and clinical data of LUAD were downloaded from the the Cancer Genome Atlas (TCGA). The immune and stromal scores were calculated for each sample using the ESTIMATE algorithm. The LUAD gene chip expression profile data and the clinical data (GSE37745, GSE11969, and GSE50081) were downloaded from Gene Expression Omnibus (GEO) for subsequent validation analysis. Differentially expressed genes were 
28

29

30

31 53

calculated between high and low score groups. Univariate Cox regression analysis was performed on differentially expressed genes (DEGs) between the two groups to obtain initial prognosis genes. These were verified by three independent LUAD cohorts from the GEO database. Multivariate Cox regression was used to identify overall survival-related DEGs. UALCAN and the Human Protein Atlas were used to analyze the mRNA/protein expression levels of the target genes. Immune cell infiltration was evaluated using the Tumor Immune Estimation Resource (TIMER) and CIBERSORT methods, and stromal cell infiltration was assessed using xCell.

Results: In this study, immune scores and stromal scores are significantly associated with the clinical characteristics of LUAD, including T stage, M stage, pathological stage, and overall survival time. 530 DEGs (18 upregulated and 512 downregulated) were found to coexist in the difference analysis with the immune scores and stromal scores subgroup. Univariate Cox regression analysis showed that 286 of the 530 DEGs were survival-related genes $(\mathrm{p}<0.05)$. Of the 286 genes initially identified, nine prognosis-related genes (CSF2RB, ITK, FLT3, CD79A, CCR4, CCR6, DOK2, AMPD1, and IGJ) were validated from three separate LUAD cohorts. In addition, functional analysis of DEGs also showed that various immunoregulatory molecular pathways, including regulation of immune response and the chemokine signaling pathways, were involved. Five genes (CCR6, ITK, CCR4, DOK2, and AMPD1) were identified as independent prognostic indicators of LUAD in specific data sets. The relationship between the expression levels of these genes and immune genes was assessed. We found that CCR6 mRNA and protein expression levels of LUAD were greater than in normal tissues. We evaluated the infiltration of immune cells and stromal cells in groups with high and low levels of expression of CCR6 in the TCGA LUAD cohort. In summary, we found a series of prognosis-related genes that were associated with the LUAD tumor microenvironment.

Keywords: Lung adenocarcinoma; Immune and stromal cells; Prognosis; tumor-infiltrating

\section{INTRODUCTION}


55

56

57

58

59

60

61

62

63

64

65

Lung cancer is a malignant disease with the highest morbidity and mortality worldwide in recent years (Bray et al. 2018). Eighty-five percent of lung cancers are non-small cell lung cancer

(NSCLC), which can be divided into lung adenocarcinomas (LUAD) and squamous carcinomas (Liu et al. 2017a). There has been an increase in the incidence of LUADs, accounting for 60\% of NSCLCs and the most common type of lung cancer (Denisenko et al.). The 5-year survival rate of patients with early-stage NSCLC after surgical treatment is 70\%-90\%; however, approximately $75 \%$ of patients lost their opportunity for surgery by the time of initial diagnosis (Goldstraw et al. 2016). Despite important advances in targeted molecular therapies and immunotherapy for lung cancer, drug resistance still limits success (Ascierto et al. 2019), and new biomarkers are warranted for different populations. This will have important implications for improving patients' early diagnosis rates and for discovering novel targeted therapies. In addition to tumor cells, stromal cells and immune cells as well as tumor-associated normal epithelial cells form malignant solid tumor tissues (Moffitt et al. 2015). Stromal cells contain cancer-related fibroblasts, pericytes, mesenchymal stem cells, blood, and lymphatic endothelial cells (Quail \& Joyce 2013). In the tumor microenvironment (TME), stromal cell components and tumor cells interact with other to establish a dynamic connection, which affects the biological function and chemical resistance of tumor cells (Joyce \& Pollard 2009). Four types of immune cells are relevant: tumor infiltrating lymphocytes (TILs), tumor-associated macrophages, myeloid-derived suppressor cells, and dendritic cells (Bedognetti et al. 2019). TILs play an important role in immune surveillance and killing of cancerous cells in patients with lung cancer (Ye et al. 2017). NK cell-mediated tumor suppressor function is closely related to tumor progression and is a key factor regulating lung cancer growth and metastasis (Fang et al. 2017). Depicting the immune infiltration of tumor microenvironment or determining the proportion of relevant normal cells in tumor tissues can help in building an accurate tumor prognosis and prediction model. Guo JY et al. demonstrated that chondroitin sulfate proteoglycan serglycin (SRGN), which is secreted by tumor cells and stromal components in the TME, promotes malignant phenotypes through interacting with tumor cell receptor CD44. Increased expression 
82

83

84

85

86

87

88

of SRGN is associated with poor prognosis in primary LUAD (Guo et al. 2017). Galectin-3 is a lectin that contributes to TME immunosuppression and regulates diverse functions. Studies suggest that increased Gal-3 expression during cancer progression augments tumor growth, invasiveness, metastatic potential, and immune suppression (Compagno et al. 2014; Farhad et al. 2018)

The quantification of various cell types allows accurate analysis of dynamically changing immune microenvironments. Experimental methods, such as single-cell sequencing (Kyrochristos et al. 2019), are expensive and cumbersome to operate; therefore, various algorithms have been developed to describe tumor immune environments, including singlesample gene set enrichment analysis (GSEA) (Foroutan et al. 2018), MCPcounter (Petitprez et al. 2018), and CIBERSORT (Chen et al. 2018). Yoshihara et al. (Yoshihara et al. 2013) proposed a new algorithm known as ESTIMATE that uses expression data to estimate the number of immune and stromal cells in malignant tumor tissues. This algorithm focuses on the formation of immune and stromal cells from major non-tumor components of tumor samples and identifies the stromal and tumor tissue-specific characteristics associated with immune cell infiltration. Its effectiveness has been shown in glioblastoma (Jia et al. 2018) and breast cancer (Winslow et al. 2016). Although accurate immune and stromal scoring of LUAD can be performed, there have been no detailed studies that have analyzed related the expression profiles with ESTIMATE. In this study, the microenvironment-related genes of the LUAD cohort of the TCGA database were studied based on the ESTIMATE score, and the prognostic genes of three different LUAD cohorts from the GEO database were verified. To further elucidate the immunological mechanisms, we assessed the role of the immune microenvironment in the development of LUAD using immune/stromal cell infiltration analysis.

\section{Materials and methods}

Database

The level 3 gene expression profile data of lung adenocarcinoma patients (using Illumina 
109

110

111

112

113

114

115

116

117

118

119

120

121

122

123

124

125

126

127

128

129

130

131

132

133

134

135

HiSeq_RNASeqV2 lung adenocarcinoma RNA expression profiles) were downloaded from the TCGA website (https://portal.gdc.cancer.gov/). The immune and stromal scores were calculated from each sample using the ESTIMATE algorithm. In addition, patient clinical data were downloaded from the TCGA official website (https://portal.gdc.cancer.gov/repository). The LUAD gene chip expression profile data and the clinical data (GSE37745, GSE11969, and GSE50081) of the samples were downloaded from the GEO official website (https://www.ncbi.nlm.nih.gov/geo/) for subsequent validation analysis. To analyze the mRNA/protein expression of CCR6 in primary LUAD and normal tissues, UALCAN (http://ualcan.path.uab.edu) was used, which was based on the TCGA database and Clinical Proteomic Tumor Analysis Consortium. To validate the expression of CCR6, the Human Protein Atlas (http://www.proteinatlas.org/) was used. Tumor Immune Estimation Resource (TIMER) (https://cistrome.shinyapps.io/timer/) was used to assess the correlation between differentially expressed gene (DEG) expression and the immune cell infiltration level. Immune cell infiltration was evaluated using the TIMER and CIBERSORT method, and stromal cell infiltration was determined using xCell (https://xcell.ucsf.edu/).

\section{Identification of differentially expressed genes (DEGs)}

The limma package in $\mathrm{R}$ was used for the analysis of differential expression genes (Ritchie et al. 2015), and the cutoff value was set to $\mid \log$ fold change $(\mathrm{FC}) \mid>1$, and a p value $<0.05$. We plotted a heat map using the cluster analysis results of DEGs in R.

\section{GO and KEGG enrichment analysis}

To investigate the molecular functions, biological processes, cell components, and signaling pathways involved in DEGs, we used the DAVID database (v6.8, https://david.ncifcrf.gov/) to perform GO analysis and KEGG pathway enrichment. p value $<0.05$ and FDR $<0.05$ were marked as significant terms. 
136

137

138

139

140

141

142

143

144

145

146

147

148

149

150

151

152

153

154

155

156

157

158

159

160

161

162

\section{Statistical analysis}

The data were assessed for normal distribution, and the central tendency was expressed as the mean \pm standard deviation for the data with normal distribution. Means were compared using a Student's t-test. Continuous variables that did not have normal distribution were analyzed using the Wilcoxon rank sum test or Kruskal-Wallis rank sum test. Student's t-tests were used to compare the distribution of scores in the high and low groups, gender, and $\mathrm{M}$ stages. The Wilcoxon rank sum test was used to compare the distribution of scores in age, $\mathrm{T}$ stages, and $\mathrm{N}$ stages. The Kruskal-Wallis rank sum test was used to compare the differences in the distribution of scores of the four different pathological stages. Spearman's rank correlation coefficient was used to measure the correlation between lung cancer stage and overall survival. Determination of survival-related genes was performed using univariate Cox regression analysis (with a $p$ value of $<0.05$ ) and the corresponding survival curves were plotted. Multivariate Cox analysis was used to evaluate the contribution of genes as independent prognosis factors for patient survival. All tests were two-tailed, and $p$ values less than 0.05 were considered statistically significant. Al analyses were performed using SPSS for Windows, software version 25.0 (SPSS Inc., Chicago, IL).

\section{RESULTS}

\section{Correlation between immune and stromal scores and clinical characteristics of LUAD}

We downloaded the gene expression profiles and clinical data of 492 patients with LUAD from the TCGA database, including 225 males (45.7\%), 267 females (54.3\%), 157 patients under 60 years of age $(31.9 \%)$, and 335 patients over 60 years of age $(68.1 \%)$. The ESTIMATE algorithm revealed stromal scores of -1959.31 to 2989.77 , and the immune scores of -1355.85 to 2905.3. The results of the relationship between the two scores and clinical data (TNM and pathological stage) are shown in Table 1. There were differences in the distribution of stromal scores between the sexes and across $\mathrm{M}$ stages. Immune scores also differed with respect to $\mathrm{T}$ and pathological stages.

We found that the immune and stromal scores of female patients were significantly higher than 
163 those of from male patients ( $\mathrm{p}=0.0069$ and 0.0049 , respectively) (Figure $1 \mathrm{~A}$ and $1 \mathrm{~B})$. The 164 immune score of T1 was significantly higher than that of T2/T3/T4 stages $(p=0.0002$, Figure 165 1C); however, but the distribution of stromal scores was not different between the two groups $166(\mathrm{p}=0.0999)$ (Figure 1D). The immune and stromal scores of the M0 stage was higher than those 167 of the M1 stage ( $\mathrm{p}=0.0098$ and 0.0366 , respectively) (Figure $1 \mathrm{E}$ and $1 \mathrm{~F}$ ). For the different 168 pathological stages of LUAD, the immune scores were different at each stage $(p=0.0276$, Figure 169 1G) and gradually decreased from stage I to stage IV (Spearman correlation coefficients: $170 \rho=-0.131, p=0.004)$; however, but the difference in stromal scores among the four 171 pathological stages was not statistically significant $(\mathrm{p}=0.1525$, Figure $1 \mathrm{H})$.

172 Next, we evaluated the correlations between immune (or stromal) scores and overall survival. 173 LUADs $(\mathrm{N}=492)$ were divided into high- and low-score groups, with 246 cases in each group. 174 Stromal scores ranged from -959.31 to 36.85 for the low group and from 39.8 to 2098.77 for the 175 high group. Immune scores ranged from -1355.85 to 952.61 for the low group and 964.93 to 2905.3 for the high group. The Kaplan-Meier survival curve analysis for immune scores

177 indicated that the median survival of the low-score group was lower than that of the high-score group (1194 d vs. 1499 d, p = 0.011; log-rank test; Figure 1I). Similarly, for stromal scores, the median survival of the low-score group was lower than that of the high-score group (1235 d vs. 1492 d, p = 0.179; log-rank test; Figure 1J), although it was not statistically significant.

\section{Comparison of gene expression profiles with LUAD immune scores and stromal scores}

We performed differential expression analysis of the high- and low- immune scoring patients and found that 884 genes were downregulated and 55 genes were upregulated (Figure 2A, Table S1). Similarly, differential analysis of the high- and low-stromal scoring groups revealed that 999 genes were downregulated and 28 genes were up-regulated $(|\log \mathrm{FC}|>1, \mathrm{p}<0.05)$ (Figure 2B, Table S1). In the Venn diagram, 18 genes were found to be upregulated in both groups (Figure 2C) and 512 genes were downregulated in both groups (Figure 2D). 
190

191

192

193

194

195

196

197

198

199

200

201

202

203

204

205

206

207

208

209

210

211

212

213

214

215

216

\section{Correlation of individual DEGs expression with overall survival and Functional} enrichment analysis of prognostic associated genes

To explore the DEGs associated with overall survival, we performed a univariate Cox regression analysis. The results (Table S2) showed that 286 of the 530 DEGs were survival-related genes $(\mathrm{p}<0.05)$. To further determine the relationship between these prognostic DEGs, we used the STRING online tool to obtain a PPI network. This network has 277 nodes and 2143 edges (Figure 3). Functional enrichment clustering of 286 prognostic DEGs was also closely related to the immune pathways. GO analysis results showed that 46 terms were statistically significant, and KEGG enrichment results showed that 22 pathways were significantly enriched $(\mathrm{p}<0.05$, FDR $<0.05)$. The top GO terms included adaptive immune response, T cell co-stimulation, MHC class II receptor activity, and transmembrane signaling receptor activity (Figure 4A). In addition, most pathways generated from the KEGG pathway enrichment analysis were associated with immune responses, such as cell adhesion molecules, primary immunodeficiency, antigen processing and presentation, and chemokine signaling pathways (Figure 4B).

\section{Further identification of prognostic genes in the GEO database}

To confirm whether the genes identified in the above steps have prognostic functions in other LUAD cohorts, we used the expression profiles and clinical data of three datasets in the GEO database for verification: GSE37745, GSE11969, and GSE50081. A total of 80 genes in GSE37745, 2703 genes in GSE11969, and 34 genes in GSE50081 were associated with survival. CSF2RB, inducible T cell kinase (ITK), and FLT3, and CD79A were found to be common among the GSE37745, GSE11969, and TCGA datasets (Figure 5A). In addition, CCR4, CCR6, DOK2, AMPD1, and IGJ were found to be common among GSE37745, GSE50081, and TCGA (Figure 5B). Kaplan-Meier survival curves were generated for the nine prognostic genes (Figure 5C). The results showed that for all but except CCR4, the overall survival time of the low expression group was significantly lower than that of the high expression group (log-rank $\mathrm{p}<0.05)$. This suggests that reduced expression of the eight genes (CSF2RB, ITK, FLT3, 
217

218

219

220

221

222

223

224

225

226

227

228

229

230

231

232

233

234

235

236

237

238

239

240

241

242

243

CD79A, CCR6, DOK2, AMPD1, and IGJ) and increased expression of one gene (CCR4) can predict poor survival of patients with for LUAD.

\section{Immune cell infiltration analysis revealed the correlation between the expression of the} DEGs and immunocytes

To confirm these findings, we used multivariate Cox regression and found that the five genes, including CCR6, ITK, CCR4, DOK2, and AMPD1, were an independent prognostic indicator for LUAD in their specific data sets (Figures 6A-6D). We investigated immune infiltration using the TIMER deconvolution approach. Interestingly, the expression of the five genes identified positively correlated to the infiltration level of different immune cells, including B cells, $\mathrm{CD}^{+}{ }^{+} \mathrm{T}$ cells, $\mathrm{CD}^{+} \mathrm{T}$ cells, macrophage cells, neutrophil cells, and dendritic cells $(p<0.05)$. The expression levels of the five genes were negatively correlated with the tumor purity $(p<0.05$; Figures 7A-7II).

\section{Evaluation of the immune and stromal status between the groups with low and high} expression of CCR6

Multivariate Cox regression analysis identified that low expression of CCR6 is an independent prognostic factor of poor survival in the TCGA cohort of 484 patients with LUAD (HR 0.29, $95 \% \mathrm{CI} 0.12-0.72, p=0.007)$. According to the results of UALCAN, mRNA and protein expression of CCR6 were all significantly upregulated in primary LUAD tissues as compared with normal samples (all $p<0.05$; Figures $8 \mathrm{~A}$ and $8 \mathrm{~B}$ ). Immunohistochemistry staining obtained from The Human Protein Atlas database also demonstrated that CCR6 was upregulated (Figures $8 \mathrm{C}$ and 8D). According to the median value of CCR6 expression levels, we divided the 484 LUAD patients into a low expression group and a high expression group. CIBERSORT and xCell were used to depict the immune cell and stromal cell landscape of CCR6 with high and low expression levels. Figures $8 \mathrm{E}$ and $8 \mathrm{~F}$ show the proportions of immune cells and stromal cells in the 484 LUAD tissues. The differential proportion of infiltration of immune and stromal cells 
244 in the group with low CCR6 expression and the group with high CCR6 expression is shown in

245 Figures 8G and 8H. CIBERSORT analysis suggests that high CCR6 expression is associated

246 with recruitment of memory B cells, resting memory CD4 T cells, monocytes, M1 macrophages,

247 resting dendritic cells, and resting mast cells (Figure S1). xCell analysis suggests that high CCR6

248 expression is associated with recruitment of adipocytes, chondrocytes, endothelial cells, and

249 fibroblasts (Figure S1). A comparison of the tumor infiltration levels among tumors with

250 different somatic copy number alterations (SCNAs) for a given gene was determined using

251 TIMER. The results showed that enrichment of the six immune cell types (B cell, CD4 ${ }^{+} \mathrm{T}$ cell,

$252 \mathrm{CD}^{+} \mathrm{T}$ cell, macrophage cell, neutrophil cell, and dendritic cell) were significantly

253 downregulated in LUAD with SCNA of CCR6 (Figure 8I).

254

255

\section{DISCUSSION}

256

257

258

259

260

261

262

263

264

265

266

267

268

269

270

Previous studies have shown that the TME can play a key role in promoting tumor progression and increasing mortality (Caetano et al. 2016; Wood et al. 2014). From the perspective of the TME, we identified a series of genes related to the overall survival of patients with LUAD based on data in the TCGA database. By analyzing differences in the overall expression profiles of patients of LUAD cases in high and low scoring groups using the ESTIMATE algorithm, we identified 286 prognostic genes involved in immune response and chemokine signaling. Importantly, we validated these results using three independent LUAD datasets from the GEO database. From the overlap among these datasets, we obtained nine prognostic genes that were associated with the immune microenvironment of LUAD. We found that five (ITK, DOK2, AMPD1, CCR4, and CCR6) out of nine genes were prognostic factors for LUAD in their specific data set.

Abnormal expression of protein tyrosine kinases (PTK) is related to tumor invasion and metastasis, tumor neovascularization, and tumor chemotherapy resistance (Knosel et al. 2014). Many drugs have been developed to target PTKs. Although tyrosine kinase inhibitors (TKIs) continue to be involved in targeted therapies for lung cancer, acquired resistance still remains a 
271 problem (Ahn et al. 2017). It has been suggested that the mechanism of action of different PTKs

272 needs additional study. IL2 inducible T cell kinase (ITK) is expressed in many immune cells. As

273 a non-receptor tyrosine kinase, it plays an important role in regulating TCRs, chemokine receptor

274 CXCR4, and FCeR-mediated signaling pathways (Sahu \& August 2009). The proteins

275 downstream of tyrosine kinase participate in the proliferation and migration of lung cancer

276 (Ghanem et al. 2014), leukemia (Tomoharu et al. 2004), gastric cancer (Liu \& Xiao 2014), and

277 other cancer cells via the receptor tyrosine kinase signaling pathway. A study has found that

278 DOK2 is a tumor suppressor gene in LUAD and that knocking out Dok2 in mice accelerates lung

279 tumorigenesis induced by oncogenic EGFR (Berger et al. 2013). We found that low expression

280 levels of ITK, and DOK2 in LUAD were associated with poor prognosis, possibly affecting

281 tumor progression via the transmembrane receptor PTK signaling pathway. However, further

282 studies are required to determine whether TKI can become a new target for lung cancer.

283 The AMPD1 gene encodes adenosine monophosphate deaminase (AMPD), a crucial enzyme in

284 purine nucleotide and energy metabolism, especially in skeletal muscle and cardiac muscle. The

285 role of AMPD1 is to catalyze the conversion of adenosine monophosphate to inosine

286 monophosphate (Smolenski et al. 2014). Xiao Luo et al. identified that AMPD1 was closely

287 associated with the survival of breast cancer patients (Luo et al. 2018). Tianzhou Zha et al. found

288 that the expression of AMPD1 in serum of patients with papillary thyroid carcinoma is closely

289 related to the malignant evolution of PTC and clinical prognosis of patients (Zha et al. 2018).

290 The relationship between AMPD1 and LUAD has not previously been elucidated.

291 Functional enrichment analysis showed that CCR6 was significantly involved in the chemokine

292 signaling pathway. This is consistent with results from previous studies that indicate that in the

293 TME, the communication among cells is dynamically regulated by a complex network of

294 immune factors, including cytokines, chemokines, and numerous growth factors (Shimizu et al.

295 2013). A variety of chemokines promote tumor proliferation, which can play a vital role in tumor

296 progression by helping tumor cells escape immune monitoring (Chang et al. 2018).

297 We found that low expression of CCR6 and high expression of CCR4 were significantly 
298

299

300

301

302

303

304

305

306

307

308

309

310

311

312

313

314

315

316

317

318

319

320

321

322

323

324

associated with poor prognosis of LUAD, based on a multi-cohort study. Multivariate Cox analysis suggests that CCR6 is an independent prognostic factor in the TCGA LUAD cohort and that CCR4 is an independent prognostic indicator in the two LUAD cohorts, GSE50081 and GSE37745. Wei Liu et al. (Liu et al. 2017b) also found that CCR4 is an independent risk factor for the overall survival of NSCLC, as it functions as a ligand for CCL17 and CCL22. CCR4 can help tumor cells escape the host immune attack by attracting Treg into the TME. Studies have identified that CCR6 can act as a ligand for CCL20 and promote lung metastasis of cancerous adrenal tissue (Raynaud et al. 2010). In a mouse model of lung cancer (Lewis lung carcinoma), expression of CCR6 in tumor cells reduced metastatic potential (Sutherland et al. 2007). Similar to our findings, a study found that increased expression of CCR6 in tumor cells is an independent predictor of a better prognosis in patients with LUAD (Minamiya et al. 2011). A recent study showed that CCL20, in coordination with CCR6, can recruit Treg cells to tumor sites (Zhang et al. 2015). To our knowledge, we have for the first time depicted the landscape of immune cell infiltration and the landscape of CCR6 in stromal cells in LUAD. Our results also suggest that there is a close correlation between CCR6 genomic alterations and immune cell enrichments in LUAD. Therefore, we hypothesized that genetic alteration of CCR6 may play an important role in LUAD. Consistent with both of these previous studies, our analysis reveals an important role of CCR6 in LUAD and its potential value as a biomarker.

\section{CONCLUSIONS}

In conclusion, using the ESTIMATE algorithm, TIMER, CIBERSORT, and xCell, we analyzed the expression profile data of patients with LUAD from the TCGA database and identified a series of genes related to the TME. Many previously unknown genes were found in addition to the genes already associated with lung cancer or immune microenvironments. Finally, we examined the interaction between CCR6 and TME. This may be important to identify the connecting mechanism between TME and LUAD prognosis. 
325

326

327

328

329

330

331

332

333

334

335

336

337

338

339

340

341

342

343

344

345

346

347

348

349

350

351

352

353

354

355

356

357

358

359

360

361

\section{ACKNOWLEDGMENTS}

This study was supported by the National Natural Science Foundation of China

(NSFC81660488) and the Guangxi Natural Science Foundation under Grant

2017GXNSFAA198123.

\section{CONFLICTS OF INTEREST}

The authors declare that there are no conflicts of interest.

\section{REFERENCES}

Ahn M-J, Sun J-M, Lee S-H, Ahn JS, and Park K. 2017. EGFR TKI combination with immunotherapy in non-small cell lung cancer. Expert opinion on drug safety 16:465-469. 10.1080/14740338.2017.1300656

Ascierto PA, Bifulco C, Buonaguro L, Emens LA, Ferris RL, Fox BA, Delgoffe GM, Galon J, Gridelli C, Merlano M, Nathan P, Odunsi K, Okada H, Paulos CM, Pignata S, Schalper KA, Spranger S, Tortora G, Zarour H, Butterfield LH, and Puzanov I. 2019. Perspectives in immunotherapy: meeting report from the "Immunotherapy Bridge 2018" (28-29 November, 2018, Naples, Italy). J Immunother Cancer 7:332. 10.1186/s40425-019-07983

Bedognetti D, Ceccarelli M, Galluzzi L, Lu R, Palucka K, Samayoa J, Spranger S, Warren S, Wong KK, Ziv E, Chowell D, Coussens LM, De Carvalho DD, DeNardo DG, Galon J, Kaufman HL, Kirchhoff T, Lotze MT, Luke JJ, Minn AJ, Politi K, Shultz LD, Simon R, Thorsson V, Weidhaas JB, Ascierto ML, Ascierto PA, Barnes JM, Barsan V, Bommareddy PK, Bot A, Church SE, Ciliberto G, De Maria A, Draganov D, Ho WS, McGee HM, Monette A, Murphy JF, Nistico P, Park W, Patel M, Quigley M, Radvanyi L, Raftopoulos H, Rudqvist NP, Snyder A, Sweis RF, Valpione S, Zappasodi R, Butterfield LH, Disis ML, Fox BA, Cesano A, and Marincola FM. 2019. Toward a comprehensive view of cancer immune responsiveness: a synopsis from the SITC workshop. J Immunother Cancer 7:131. 10.1186/s40425-019-0602-4

Berger AH, Ming C, Alessandro M, Janas JA, Masaru N, Bronson RT, Taylor BS, Marc L, Linda VA, and Katerina P. 2013. DOK2 Inhibits EGFR-Mutated Lung Adenocarcinoma. Plos One 8:e79526.

Bray F, Ferlay J, Soerjomataram I, Siegel RL, Torre LA, and Jemal A. 2018. Global cancer statistics 2018: GLOBOCAN estimates of incidence and mortality worldwide for 36 cancers in 185 countries. CA: A Cancer Journal for Clinicians.

Caetano MS, Zhang H, Cumpian AM, Gong L, Unver N, Ostrin EJ, Daliri S, Chang SH, Ochoa CE, Hanash S, Behrens C, Wistuba, II, Sternberg C, Kadara H, Ferreira CG, Watowich SS, and Moghaddam SJ. 2016. IL6 Blockade Reprograms the Lung Tumor Microenvironment 
362

363

364

365

366

367

368

369

370

371

372

373

374

375

376

377

378

379

380

381

382

383

384

385

386

387

388

389

390

391

392

393

394

395

396

397

398

399

400

401

402 to Limit the Development and Progression of K-ras-Mutant Lung Cancer. Cancer Res 76:3189-3199. 10.1158/0008-5472.Can-15-2840

Chang WA, Yen MC, Hung JY, Yang CJ, Jian SF, Yeh IJ, Liu KT, Hsu YL, and Kuo PL. 2018. Investigation of the role of tumor necrosis factor-like weak inducer of apoptosis in nonsmall cell lung cancer. Oncology Reports 39:573-581.

Chen B, Khodadoust MS, Liu CL, Newman AM, and Alizadeh AA. 2018. Profiling Tumor Infiltrating Immune Cells with CIBERSORT. Methods in molecular biology (Clifton, $\mathrm{NJ}$ ) 1711:243-259. 10.1007/978-1-4939-7493-1_12

Compagno D, Gentilini LD, Jaworski FM, Perez IG, Contrufo G, and Laderach DJJG. 2014. Glycans and galectins in prostate cancer biology, angiogenesis and metastasis. 24:899906.

Denisenko TV, Budkevich IN, Zhivotovsky BJCD, and Disease. Cell death-based treatment of lung adenocarcinoma. 9:117.

Fang F, Xiao W, and Tian Z. 2017. NK cell-based immunotherapy for cancer. Semin Immunol 31:37-54. 10.1016/j.smim.2017.07.009

Farhad M, Rolig AS, and Oncoimmunology WLRJ. 2018. The role of Galectin-3 in modulating tumor growth and immunosuppression within the tumor microenvironment. 7:-.

Foroutan M, Bhuva DD, Lyu R, Horan K, Cursons J, and Davis MJ. 2018. Single sample scoring of molecular phenotypes. BMC Bioinformatics 19:404. 10.1186/s12859-018-2435-4

Ghanem T, Bracken J, Kasem A, Wen GJ, and Mokbel K. 2014. mRNA expression of DOK1-6 in human breast cancer. World Journal of Clinical Oncology 5:156-163.

Goldstraw P, Chansky K, Crowley J, Rami-Porta R, Asamura H, Eberhardt WE, Nicholson AG, Groome P, Mitchell A, and Bolejack V. 2016. The IASLC Lung Cancer Staging Project: Proposals for Revision of the TNM Stage Groupings in the Forthcoming (Eighth) Edition of the TNM Classification for Lung Cancer. $J$ Thorac Oncol 11:39-51. 10.1016/j.jtho.2015.09.009

Guo JY, Hsu HS, Tyan SW, Li FY, Shew JY, Lee WH, and Chen JY. 2017. Serglycin in tumor microenvironment promotes non-small cell lung cancer aggressiveness in a CD44dependent manner. Oncogene 36:2457-2471. 10.1038/onc.2016.404

Jia D, Li S, Li D, Xue H, Yang D, and Liu Y. 2018. Mining TCGA database for genes of prognostic value in glioblastoma microenvironment. Aging (Albany NY) 10:592-605. 10.18632/aging.101415

Joyce JA, and Pollard JW. 2009. Microenvironmental regulation of metastasis. Nat Rev Cancer 9:239-252. 10.1038/nrc2618

Knosel T, Kampmann E, Kirchner T, and Altendorf-Hofmann A. 2014. [Tyrosine kinases in soft tissue tumors]. Pathologe 35 Suppl 2:198-201. 10.1007/s00292-014-1958-3

Kyrochristos ID, Ziogas DE, Goussia A, Glantzounis GK, and Roukos DH. 2019. Bulk and SingleCell Next-Generation Sequencing: Individualizing Treatment for Colorectal Cancer. Cancers (Basel) 11. 10.3390/cancers11111809

Liu G, Pei F, Yang F, Li L, Amit A, Liu S, Buchan J, and William CJIJoMS. 2017a. Role of Autophagy and Apoptosis in Non-Small-Cell Lung Cancer. 18:367-. 
403

404

405

406

407

408

409

410

411

412

413

414

415

416

417

418

419

420

421

422

423

424

425

426

427

428

429

430

431

432

433

434

435

436

437

438

439

440

441

442

443

Liu HS, and Xiao HS. 2014. MicroRNAs as potential biomarkers for gastric cancer. World Journal of Gastroenterology 20:12007-12017.

Liu W, Wei X, Li L, Wu X, Yan J, Yang H, and Song F. 2017b. CCR4 mediated chemotaxis of regulatory $\mathrm{T}$ cells suppress the activation of T cells and NK cells via TGF- $\beta$ pathway in human non-small cell lung cancer. Biochemical \& Biophysical Research Communications 488:196.

Luo X, Yu H, Song Y, and Physiology TSJJoC. 2018. Integration of metabolomic and transcriptomic data reveals metabolic pathway alteration in breast cancer and impact of related signature on survival. 234:13021-13031.

Minamiya Y, Saito H, Takahashi N, Ito M, Toda H, Ono T, Konno H, Motoyama S, and Ogawa JIJTB. 2011. Expression of the chemokine receptor CCR6 correlates with a favorable prognosis in patients with adenocarcinoma of the lung. 32:197-202.

Moffitt RA, Marayati R, Flate EL, Volmar KE, Loeza SG, Hoadley KA, Rashid NU, Williams LA, Eaton SC, Chung AH, Smyla JK, Anderson JM, Kim HJ, Bentrem DJ, Talamonti MS, Iacobuzio-Donahue CA, Hollingsworth MA, and Yeh JJ. 2015. Virtual microdissection identifies distinct tumor- and stroma-specific subtypes of pancreatic ductal adenocarcinoma. Nat Genet 47:1168-1178. 10.1038/ng.3398

Petitprez F, Vano YA, Becht E, Giraldo NA, de Reyniès A, Sautès-Fridman C, and Fridman WH. 2018. Transcriptomic analysis of the tumor microenvironment to guide prognosis and immunotherapies. Cancer immunology, immunotherapy : CII 67:981-988. $10.1007 / \mathrm{s} 00262-017-2058-\mathrm{z}$

Quail DF, and Joyce JA. 2013. Microenvironmental regulation of tumor progression and metastasis. Nat Med 19:1423-1437. 10.1038/nm.3394

Raynaud CM, Olaf M, Philippe D, Frédéric C, Ken André O, Vincent DM, Fabrice A, Laure S, and Jean-Charles S. 2010. Expression of chemokine receptor CCR6 as a molecular determinant of adrenal metastatic relapse in patients with primary lung cancer. Clinical Lung Cancer 11:187-191.

Ritchie ME, Belinda P, Di W, Yifang H, Law CW, Wei S, and Smyth GK. 2015. limma powers differential expression analyses for RNA-sequencing and microarray studies. Nucleic Acids Research 43:e47.

Sahu N, and August A. 2009. ITK inhibitors in inflammation and immune-mediated disorders. Curr Top Med Chem 9:690-703. 10.2174/156802609789044443

Shimizu K, Okita R, and Nakata M. 2013. Clinical significance of the tumor microenvironment in non-small cell lung cancer. Ann Transl Med 1:20. 10.3978/j.issn.2305-5839.2013.06.01

Smolenski RT, Rybakowska I, Turyn J, Romaszko P, Zabielska M, Taegtmeyer A, Słomińska EM, Kaletha KK, and Barton PJ. 2014. AMP deaminase 1 gene polymorphism and heart disease-a genetic association that highlights new treatment. Cardiovasc Drugs Ther 28:183-189. 10.1007/s 10557-013-6506-5

Sutherland A, ., J-F M, Maho A, ., and Parmentier M, . 2007. Expression of the chemokine receptor CCR6 in the Lewis lung carcinoma (LLC) cell line reduces its metastatic potential in vivo. Cancer Gene Therapy 14:847-857. 
444 Tomoharu Y, Masaki S, Atsushi I, Asuka I, Yasuhiro E, Mitsujiro O, Kazuho H, Hisaaki S,

445

446

447

448

449

450

451

452

453

454

455

456

457

458

459

460

461

462

463

464

465

466

467

468

469

470

471

472

473

474

475

476

477 Katsuko S, and Kohichiro T. 2004. Role of Dok-1 and Dok-2 in myeloid homeostasis and suppression of leukemia. Journal of Experimental Medicine 200:1681-1687.

Winslow S, Lindquist KE, Edsjo A, and Larsson C. 2016. The expression pattern of matrixproducing tumor stroma is of prognostic importance in breast cancer. BMC Cancer 16:841. 10.1186/s12885-016-2864-2

Wood SL, Pernemalm M, Crosbie PA, and Whetton AD. 2014. The role of the tumormicroenvironment in lung cancer-metastasis and its relationship to potential therapeutic targets . Cancer Treatment Reviews 40:558-566.

Ye L, Zhang F, Li H, Yang L, Lv T, Gu W, and Song Y. 2017. Circulating Tumor Cells Were Associated with the Number of T Lymphocyte Subsets and NK Cells in Peripheral Blood in Advanced Non-Small-Cell Lung Cancer. Dis Markers 2017:5727815. $10.1155 / 2017 / 5727815$

Yoshihara K, Shahmoradgoli M, Martínez E, Vegesna R, Kim H, Torresgarcia W, Treviño V, Shen H, Laird PW, and Levine DA. 2013. Inferring tumour purity and stromal and immune cell admixture from expression data. Nature Communications 4:2612.

Zha T, Wu HJE, and Medicine T. 2018. Expression of serum AMPD1 in thyroid carcinoma and its clinical significance.

Zhang CY, Qi Y, Li XN, Yang Y, Liu DL, Zhao J, Zhu DY, Wu K, Zhou XD, Zhao SJB, and Pharmacotherapy. 2015. The role of CCL20/CCR6 axis in recruiting Treg cells to tumor sites of NSCLC patients. 69:242-248.

\section{FIGURE LEGENDS}

Figure 1. Relationships among immune and stromal scores, clinical characteristics, and overall survival.

(A-H) Distribution of stromal and immune scores based on gender, T stage, $\mathrm{M}$ stage, and pathological stage. (I, J) Kaplan-Meier survival analysis of low and high score groups based on immune and stromal scores. $(* \mathrm{p}<0.05, * * \mathrm{p}<0.01, * * * \mathrm{p}<0.001)$

Figure 2. Comparison of gene expression profiles with immune scores and stromal scores in LUAD.

(A, B) Heatmaps were drawn for the DEGs obtained from the respective high and low group comparisons of the immune scores and stromal scores. $(C, D)$ Venn diagrams drawn from the DEGs that are up-regulated or down-regulated in immune scores and stromal scores. 
Figure 3. PPI network of 286 prognostic DEGs.

480 
505 lung adenocarcinoma.

506 (O-U) The TIMER database showed that CCR4 expression was negatively correlated with the

507 tumor purity and was positively correlated with the infiltration of six types of immune cells in

508 lung adenocarcinoma.

509 (V-BB) The TIMER database showed that AMPD1 expression was negatively correlated with

510 the tumor purity and was positively correlated with the infiltration of six types of immune cells

511 in lung adenocarcinoma.

512 (CC-II) The TIMER database showed that DOK2 expression was negatively correlated with the

513 tumor purity and was positively correlated with the infiltration of six types of immune cells in

514 lung adenocarcinoma.

515

516 Figure 8. mRNA and protein expression and immune and stromal status of CCR6 in

517 LUAD.

518 (A-B) mRNA and protein expression of CCR6 in primary LUAD tissues as compared with 519 normal samples (UALCAN). (C-D) CCR6 protein was not expressed in normal lung tissues, 520 whereas high expression was observed in LUAD tissues (The Human Protein Atlas). (E-F) Bar

521 charts of 22 immune cell proportions (CIBERSORT) and 14 stromal cell proportions (xCell)

522 between the groups with low and high expression of CCR6 in LUAD. (G-H) Differential

523 expression of different types of immune and stromal cells between the groups with low and high

524 expression of CCR6 in LUAD. (I) The association of SCNAs of CCR6 with immune infiltration

525 in LUAD (TIMER).

526

527 TABLE LEGENDS

528 Table 1. The relationship between the two scores and clinical characteristics.

529

\section{SUPPORTING INFORMATION}

531 Table S1. List of DEGs in the immune score group and the stromal score group.

532 Table S2. 286 survival-related genes were analyzed using univariate Cox regression. 
533 Figure S1. Comparative expression of immune cells and stromal cells between the groups with 534 low and high expression of CCR6 in LUAD. 


\section{Figure 1}

Relationships among immune and stromal scores, clinical characteristics, and overall survival.

Distribution of stromal and immune scores based on gender (A), T stage (B), M stage (C), and pathological stage (D) . Kaplan-Meier survival analysis of low and high score groups based on stromal (E) and immune scores (F). $(* p<0.05, * * p<0.01, * * * p<0.001)$ 

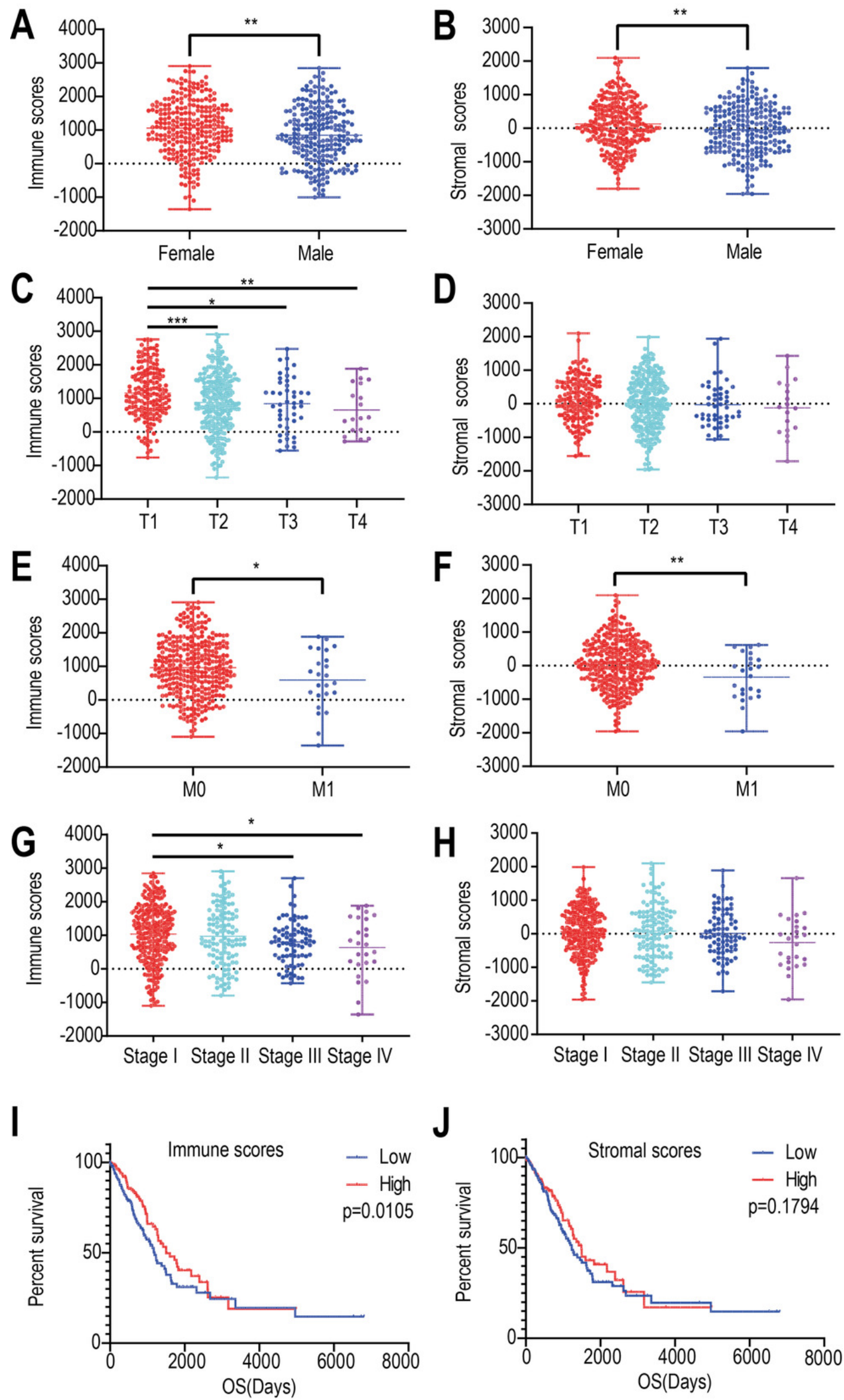

Peer) reviewing PDF | (2020:01:45176:2:0:NEW 14 Jun 2020) 
Figure 2

Comparison of gene expression profiles with immune scores and stromal scores in LUAD.

Heatmaps were drawn for the DEGs obtained from the respective high and low group comparisons of the immune scores (A) and stromal scores (B). Venn diagrams drawn from the DEGs that are up- or down-regulated in immune scores (C) and stromal scores (D). 
A Immune scores

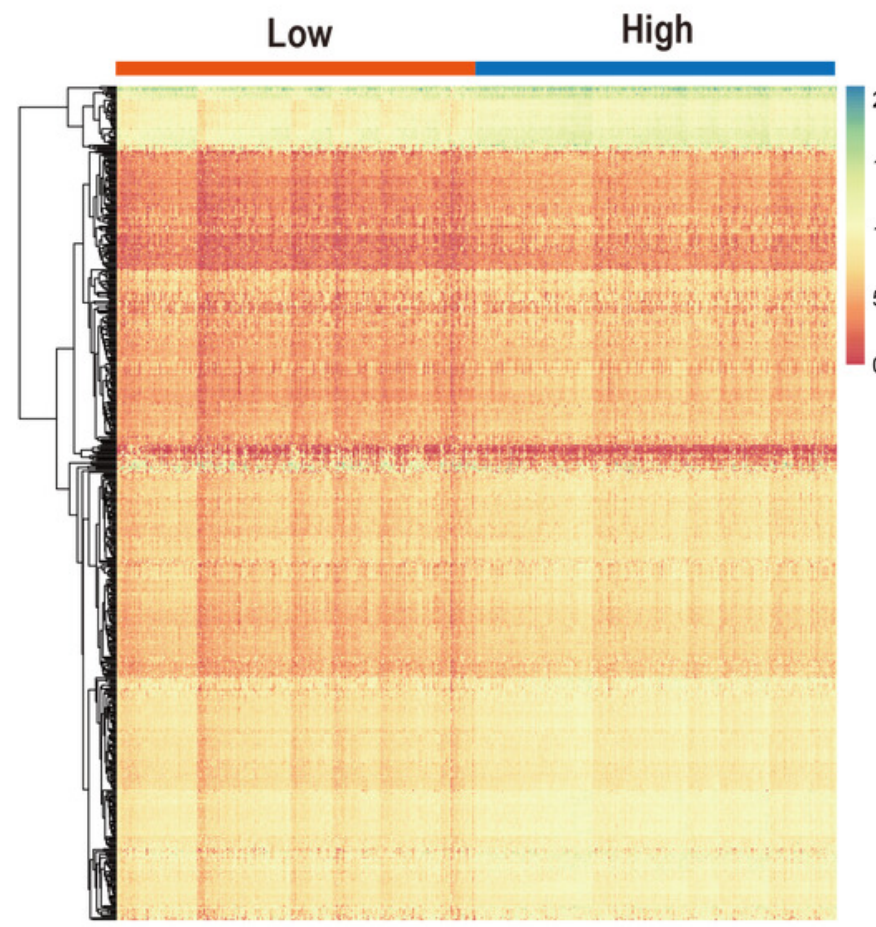

C Up-regulated DEGs in stromal score group

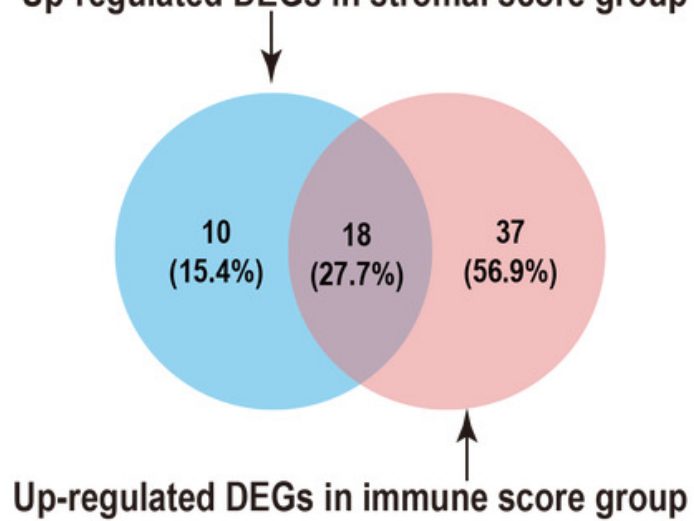

B

\section{Stromal scores}

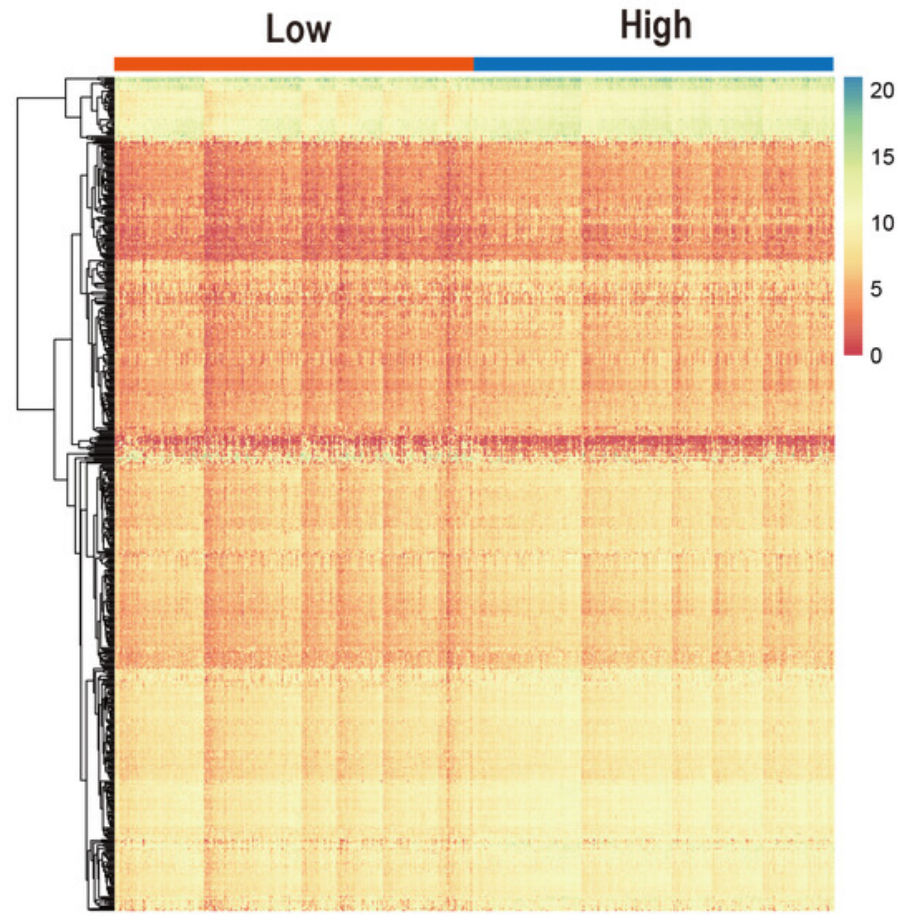

D Down-regulated DEGs in stromal score group

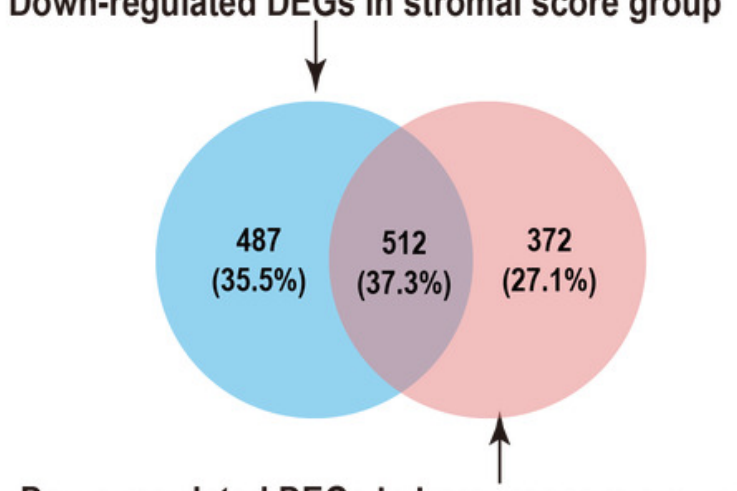

Down-regulated DEGs in immunescore group 
Figure 3

PPI network of 286 prognostic DEGs.

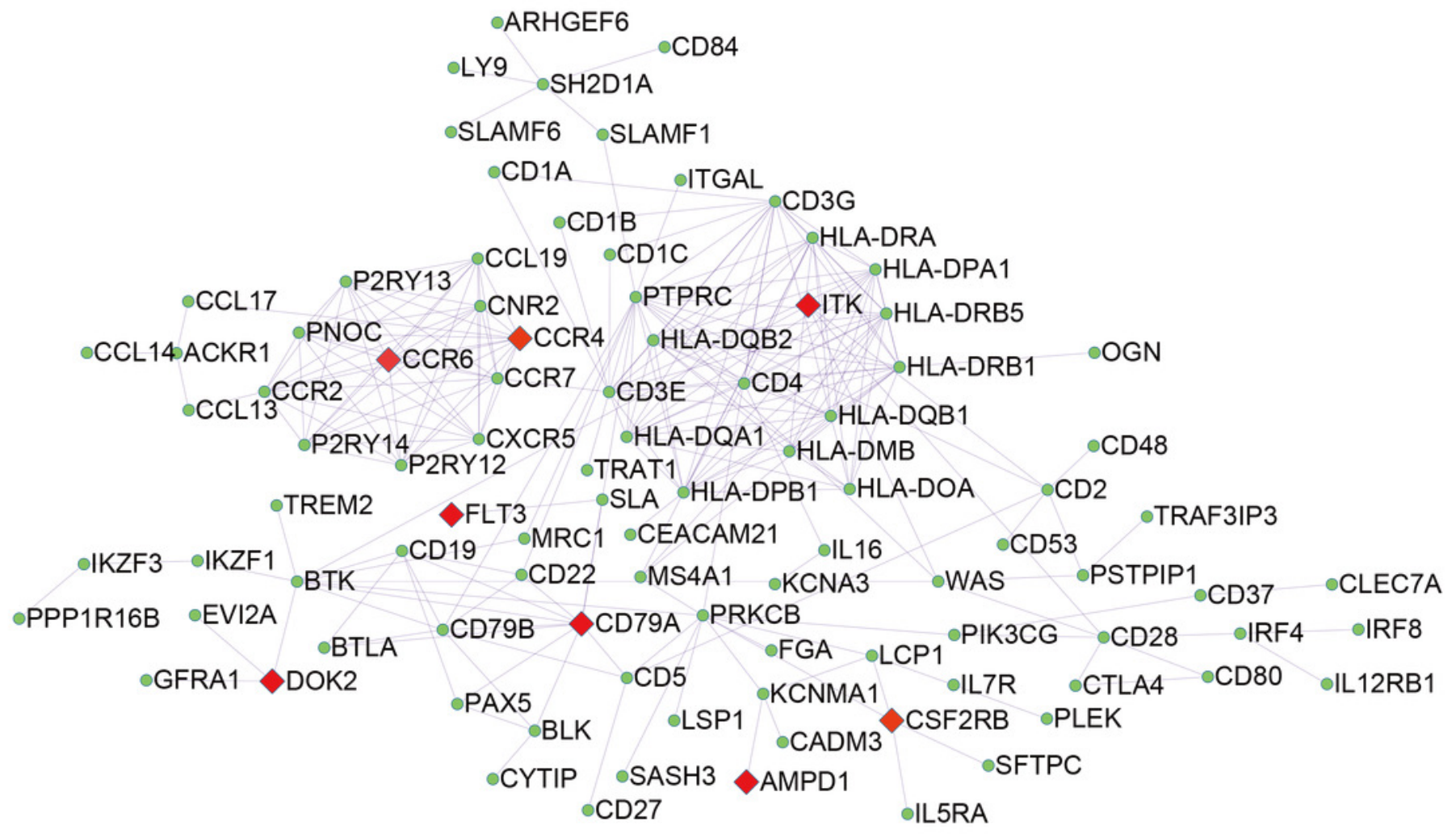




\section{Figure 4}

GO term and KEGG analysis of 286 DEGs.

(A) GO analysis of 286 DEGs . (B) KEGG enrichments of 286 DEGs. GO - gene ontology; KEGG

- Kyoto Encyclopedia of Genes and Genomes; MF - molecular function; CC - cellular component; BP - biological process.

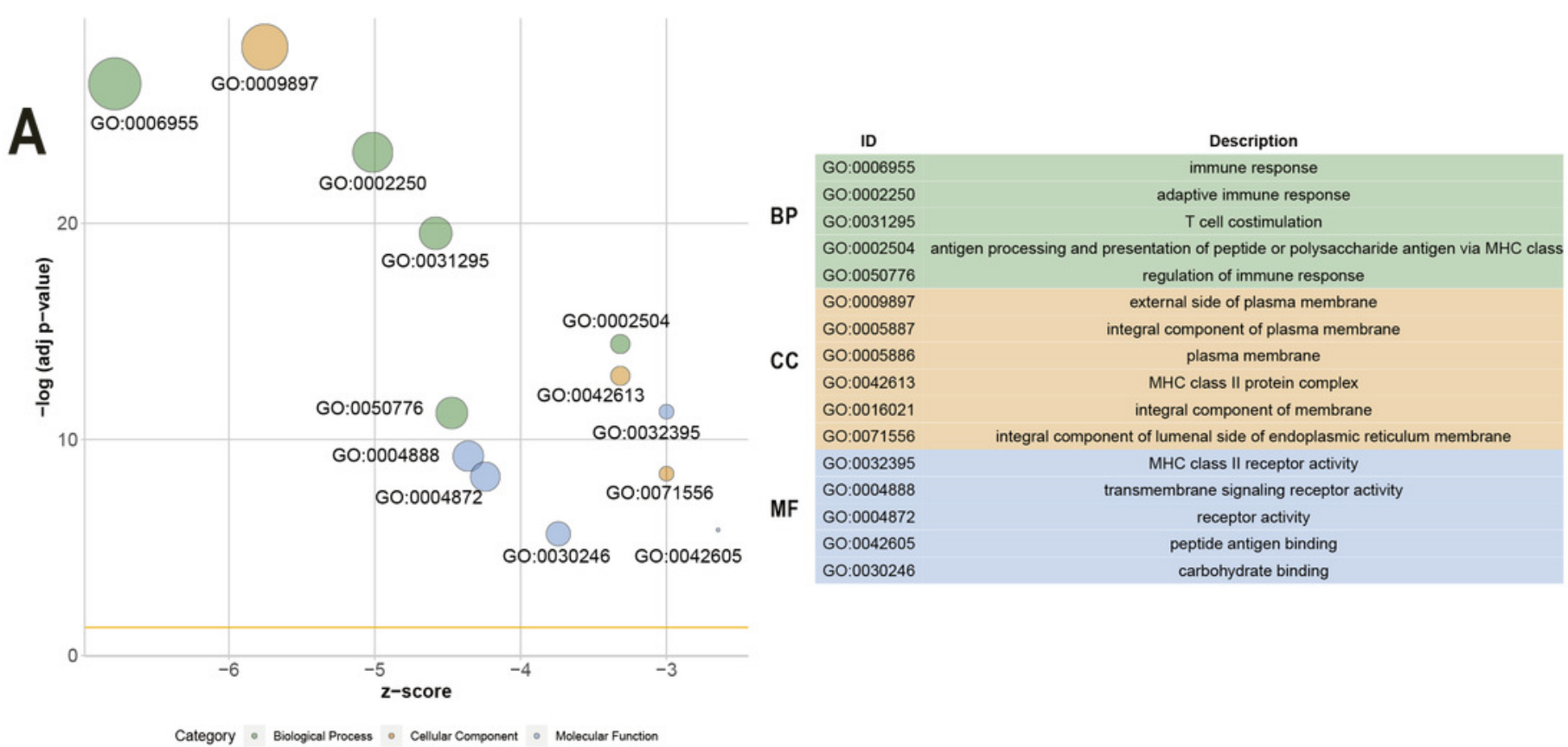

B

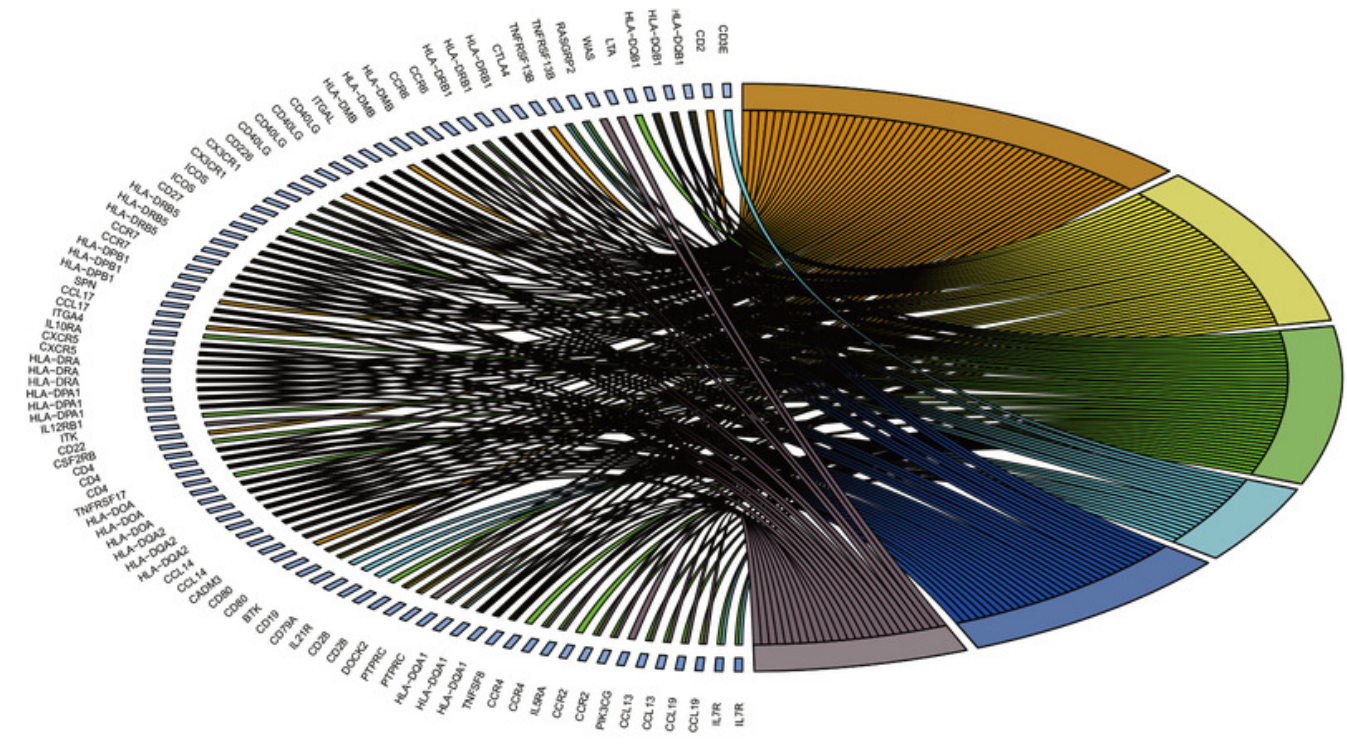




\section{Figure 5}

Correlation between expression of nine prognosis-related genes and overall survival in GEO.

(A) The Venn diagram shows prognosis-related genes based on GSE11969, GSE37745, and TCGA cross-validation. (B) The Venn diagram shows prognosis-related genes based on GSE50081, GSE37745, and TCGA cross-validation. (C) Kaplan-Meier survival curves were generated for nine prognosis genes (AMP11, CCR4, CCR6, CD79A, CSF2RB, DOK2, FLT3, IG), and ITK) extracted from the comparison of groups of high (red line) and low (blue line) gene expression. 
A

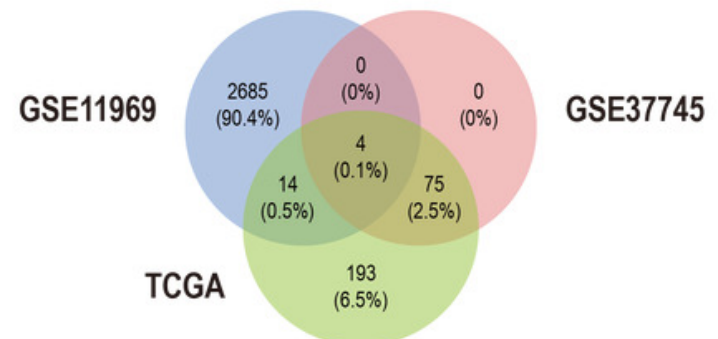

C
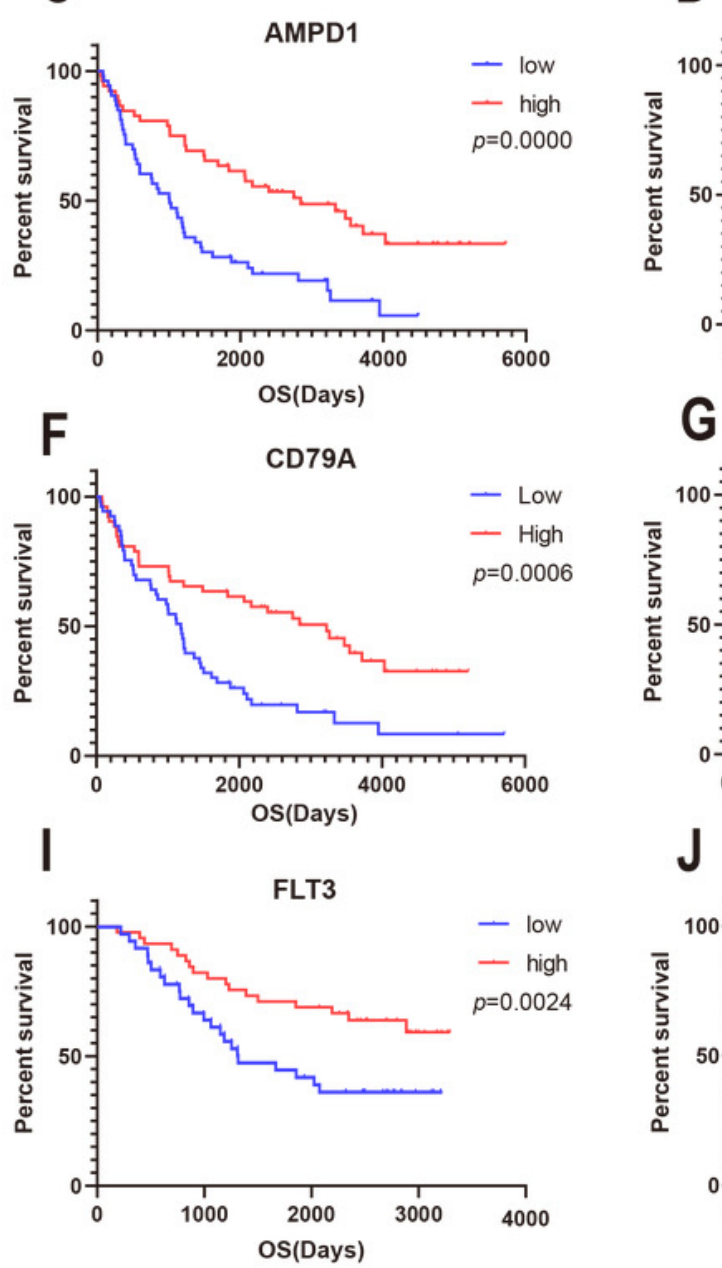

B

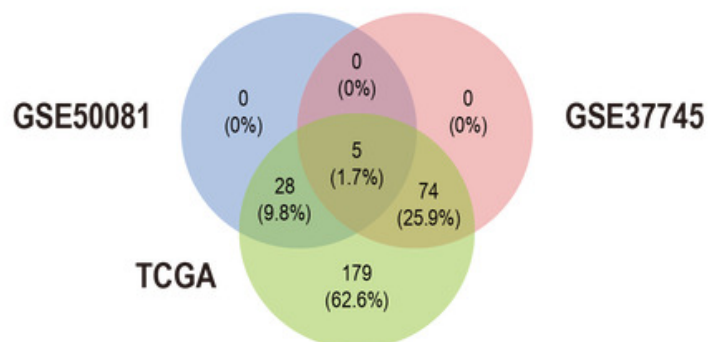

E
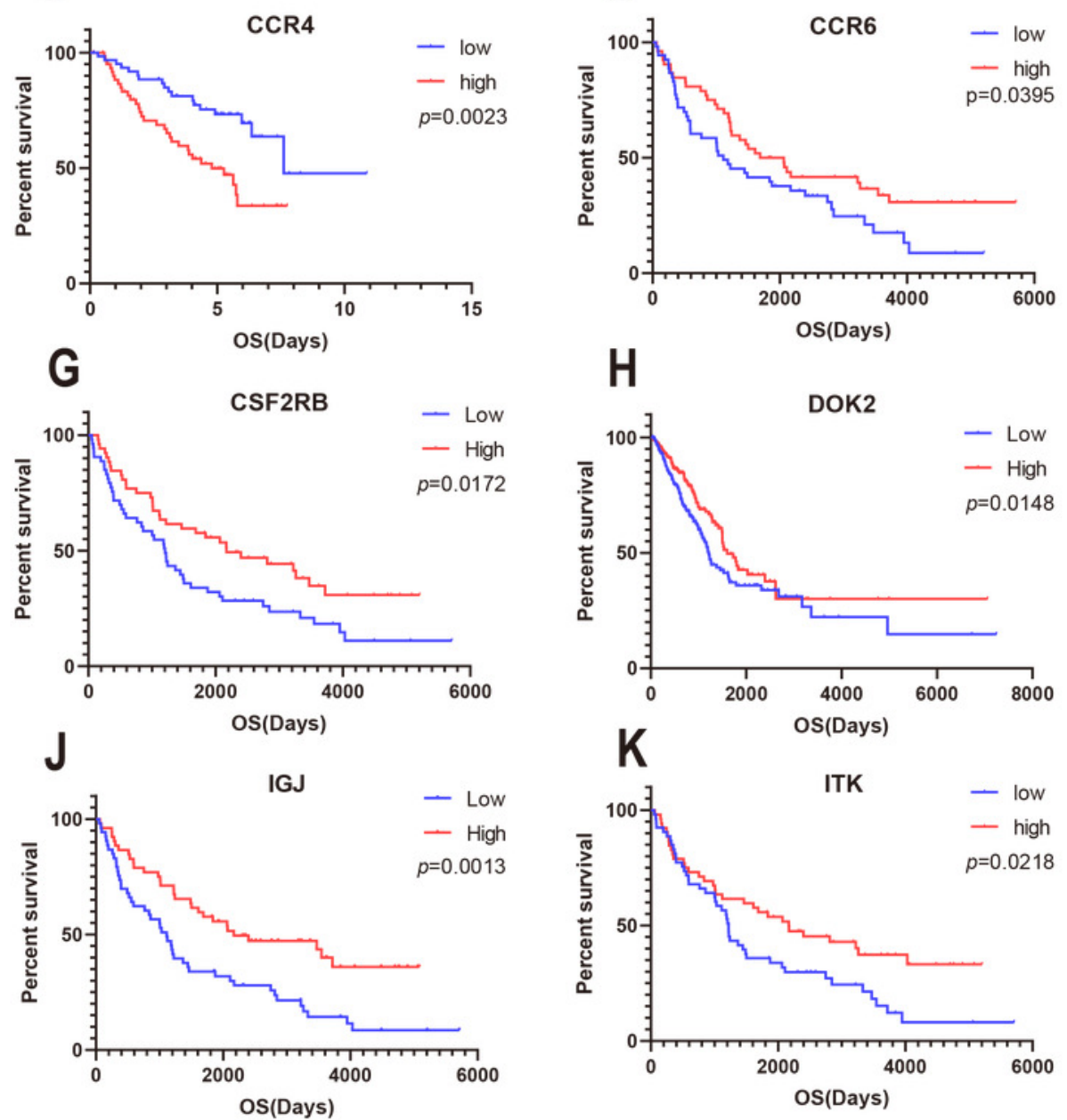
Figure 6

The multivariate Cox regression analysis of 9 prognostic genes.

The HR and P-value from the multivariate Cox regression of selected prognostic genes in TCGA (A), GSE11969 (B), GSE37745 (C), GSE50081 (D). (*p < 0.05, ** $p<0.01$ )

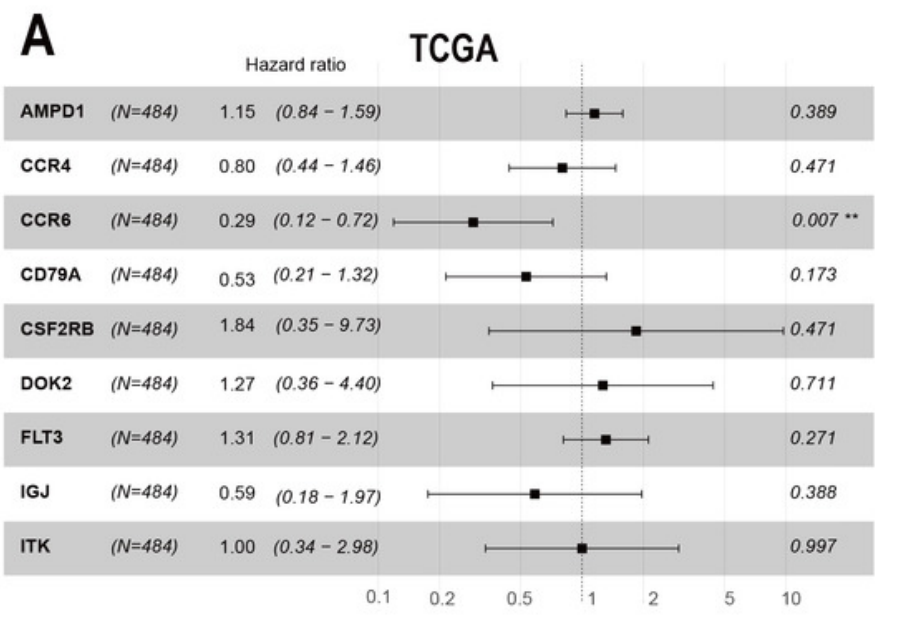
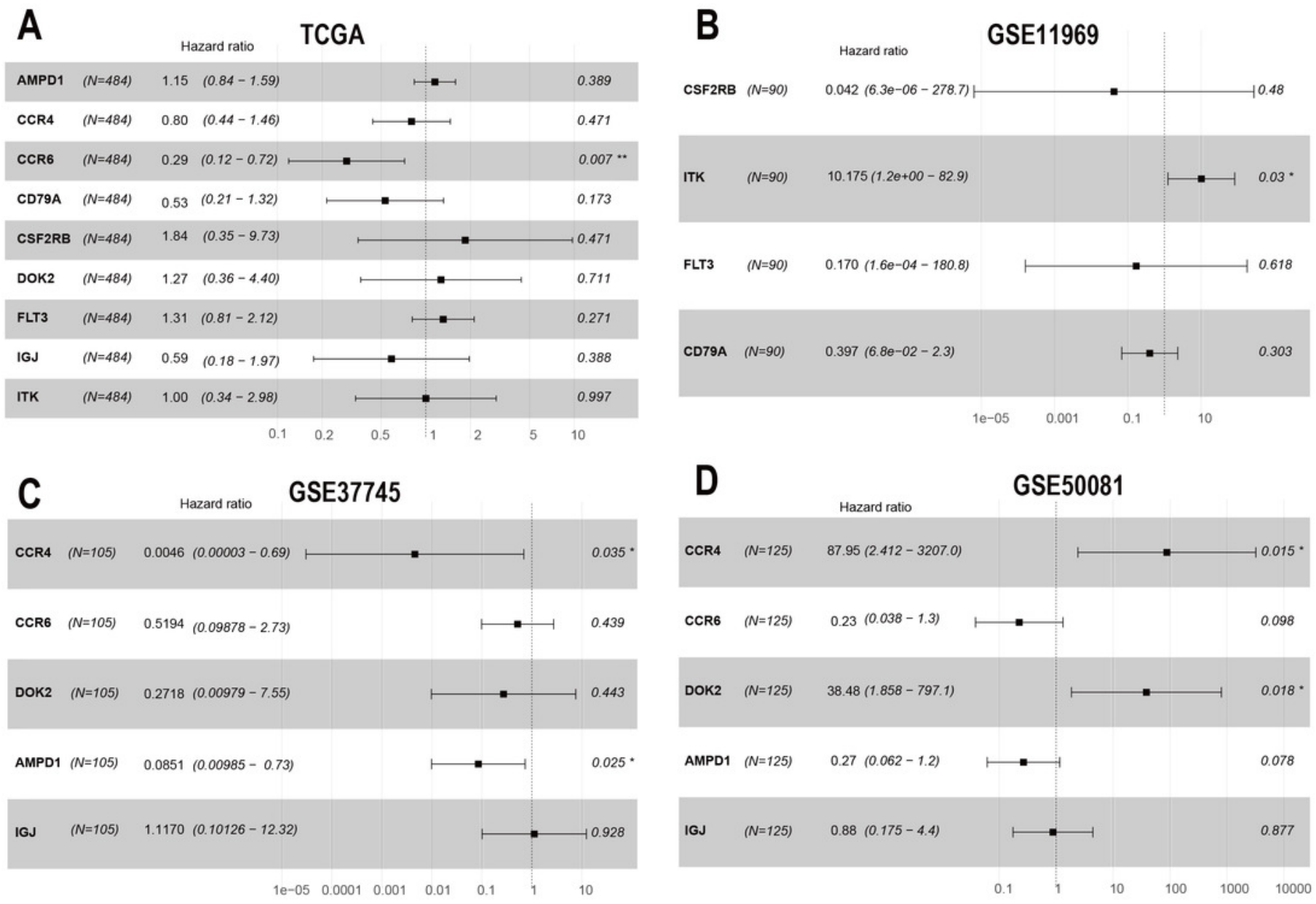


\section{Figure 7}

Correlation analysis between the expressions of mainly identified DEGs (CCR6, ITK, CCR4, DOK2, AMPD1) and infiltration levels of B cell, CD8+ T cell, CD4+ T cell, macrophage, neutrophil, and dendritic cell.
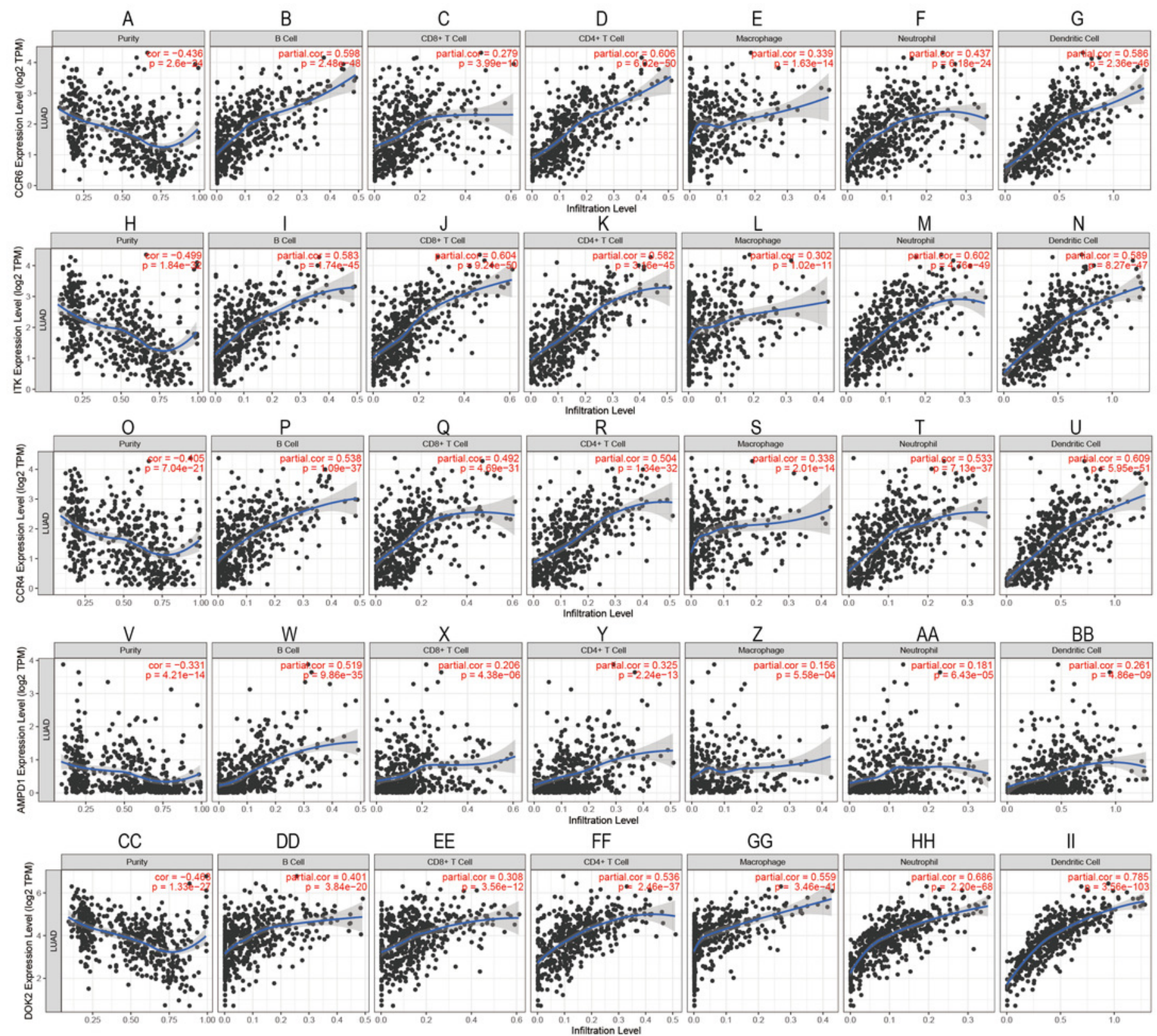


\section{Figure 8}

mRNA and protein expressions, immune and stromal status of CCR6 in LUAD.

(A) mRNA and protein expressions of CCR6 in primary LUAD tissues compared to normal samples (UALCAN). (B) CCR6 protein were not expressed in normal lung tissues, whereas its high expressions were observed in LUAD tissues (The Human Protein Atlas). (C) Bar charts of 22 immune cell proportions (CIBERSORT) and 14 stromal cell proportions (xCell) between low and high expression of CCR6 in LUAD. (D) Differential expression of different types of immune cells and stromal cells between low and high expression of CCR6 in LUAD . (E) The association of SCNAs of CCR6 with immune infiltration in LUAD (TIMER). 
A

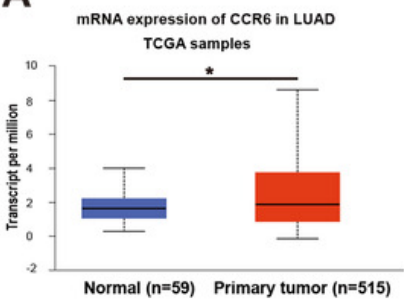

E

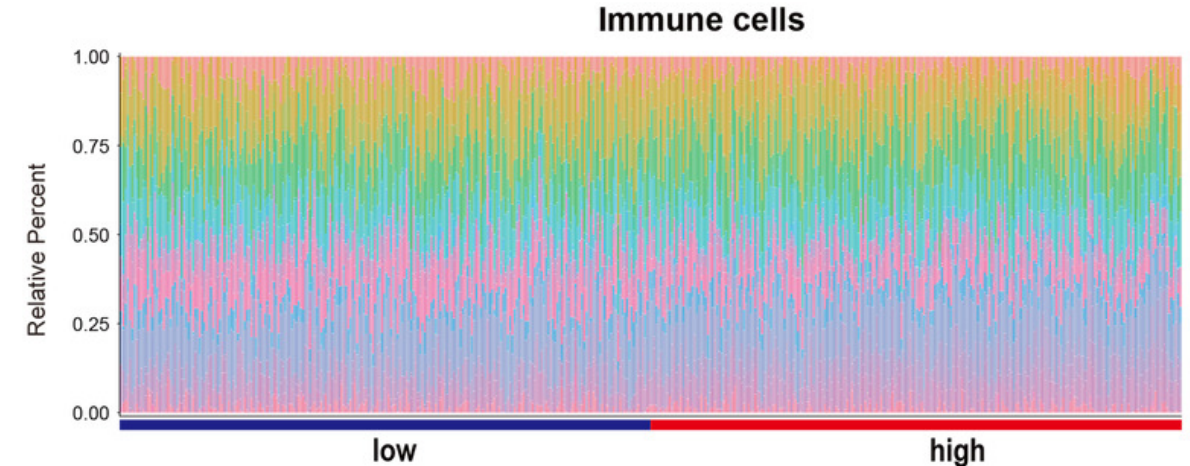

G

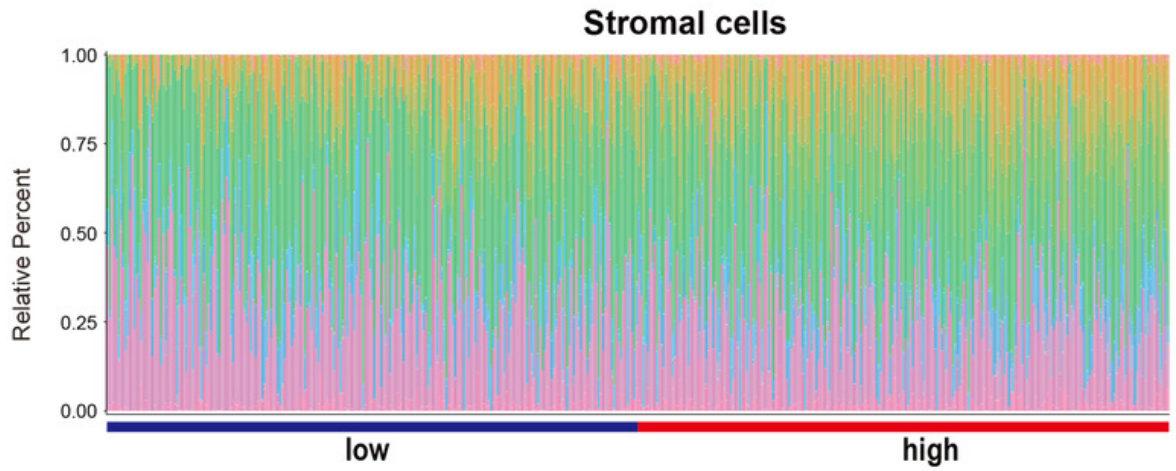

C

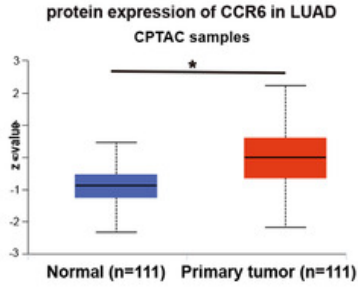

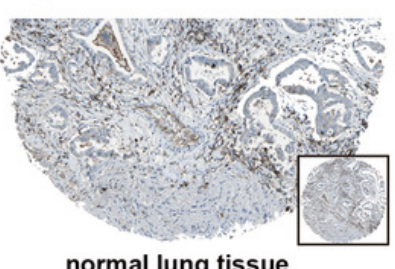

normal lung tissue
Immune cells

\section{F}

G
D

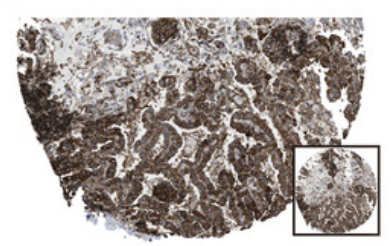

LUAD tissue
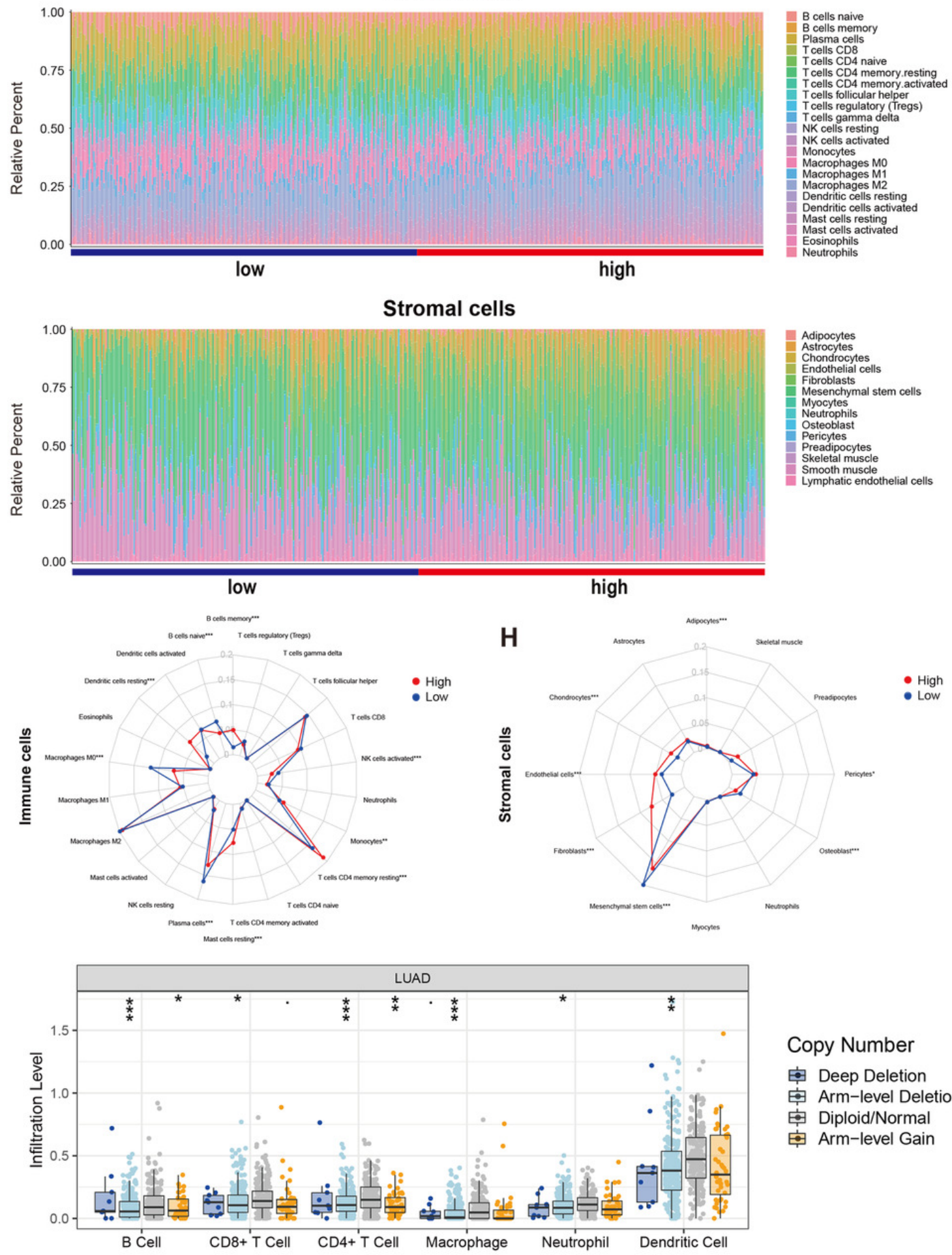

Copy Number

审 Deep Deletion

审 Arm-level Deletion

审 Diploid/Normal

审 Arm-level Gain 


\section{Table $\mathbf{1}$ (on next page)}

The relationship between the two scores and clinical characteristics. 
Table 1. The relationship between the two scores and clinical characteristics.

\begin{tabular}{|c|c|c|c|c|c|}
\hline Variable & Patients & Stromal scores & $\mathrm{P}$ value & Immune scores & $\mathrm{P}$ value \\
\hline Scores & & & $<0.000 * *$ & & $<0.000 * *$ \\
\hline Low & 246 & $-539.40 \pm 27.69$ & & $490.60 \pm 45.44$ & \\
\hline High & 246 & $635 \pm 26.75$ & & $1437 \pm 41.31$ & \\
\hline Age, years & & & 0.223 & & 0.100 \\
\hline$\leq 60$ & 157 & 235.10 & & 231.1 & \\
\hline$>60$ & 335 & 251.84 & & 253.72 & \\
\hline Sex & & & $0.007 * *$ & & $0.005 * *$ \\
\hline Male & 225 & $-48.34 \pm 48.91$ & & $849.60 \pm 55.64$ & \\
\hline Female & 267 & $128.80 \pm 43.52$ & & $1060 \pm 49.79$ & \\
\hline T stage & & & 0.101 & & $0.001 * *$ \\
\hline $\mathrm{T} 1$ & 167 & 260.99 & & 277 & \\
\hline $\mathrm{T} 2 / \mathrm{T} 3 / \mathrm{T} 4$ & 325 & 238.85 & & 230.40 & \\
\hline N stage & & & 0.768 & & 0.155 \\
\hline No & 318 & 242.34 & & 247.45 & \\
\hline N1/N2/N3 & 163 & 238.39 & & 228.42 & \\
\hline M stage & & & $0.010 * *$ & & $0.037^{*}$ \\
\hline M0 & 324 & $54.67 \pm 40.45$ & & $961.70 \pm 46.01$ & \\
\hline M1 & 24 & $-343.60 \pm 137.40$ & & $592.90 \pm 177.2$ & \\
\hline Stage & & & 0.137 & & $0.023^{*}$ \\
\hline I & 262 & 249.35 & & 256.45 & \\
\hline II & 118 & 246.36 & & 242.92 & \\
\hline III & 79 & 232.61 & & 210.16 & \\
\hline IV & 25 & 183.72 & $\square$ & 196.48 & $\square$ \\
\hline
\end{tabular}

Tips: $\mathrm{p}<0.05^{*} ; \mathrm{p}<0.01 * *$ 